Musées, Patrimoine et Culture scientifiques et techniques

\title{
Théorie et pratique du son dans les musées
}

\section{Yann Laville}

URL : http://journals.openedition.org/ocim/1530

DOI : $10.4000 /$ ocim. 1530

ISSN : 2108-646X

\section{Éditeur}

OCIM

Édition imprimée

Date de publication : 1 mai 2015

Pagination : 43-46

ISSN : 0994-1908

Référence électronique

Yann Laville, "Théorie et pratique du son dans les musées », La Lettre de l'OC/M [En ligne], 159 | 2015, mis en ligne le 01 mai 2016, consulté le 20 avril 2019. URL : http://journals.openedition.org/ocim/1530 ; DOI : 10.4000/ocim. 1530

Ce document a été généré automatiquement le 20 avril 2019.

Tous droits réservés 


\title{
Théorie et pratique du son dans les musées
}

\author{
Yann Laville
}

Dans son article Luc Martinez s'interrogeait sur les possibilités techniques offertes par les outils numériques pour la sonorisation des musées et expositions et plaidait pour une reconnaissance du son comme composante du projet muséographique.

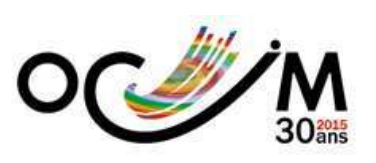

Oyez ! Le son s'expose !

Luc Martinez *

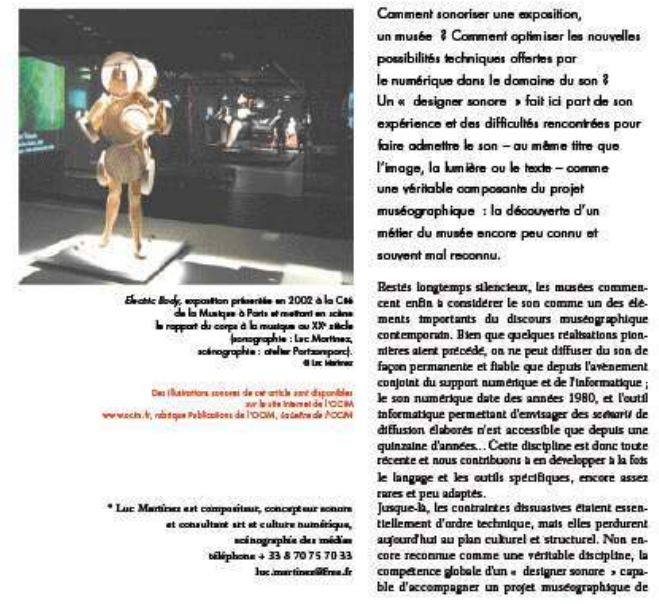

Revenir sur l'article de Luc Martinez Oyez! Le son s'expose! neuf ans plus tard, offre une occasion passionnante de mesurer le décalage qui s'instaure invariablement entre progrès techniques, imaginaires que leurs applications nourrissent et usages réels dans 
les institutions culturelles de type musée. Comme cette dialectique fut au centre des expositions présentées au musée d'Ethnographie de Neuchâtel (MEN) depuis 2006 (plus particulièrement Bruits du 2 octobre 2010 au 15 septembre 2011 et Hors-Champs du 3 novembre 2012 au 29 décembre 2013), je me permettrai de fonder ma réflexion sur ces exemples et les recherches qui les ont accompagnées. Le but n'est aucunement de comparer ce qui n'est pas comparable et encore moins de hiérarchiser méthodes ou résultats mais d'interroger le hiatus entre théorie et pratique de l'intégration sonore en vertu des contraintes propres à une institution de taille moyenne, ne bénéficiant pas des budgets alloués aux expositions des grands musées métropolitains.

2 Pour entrer dans le vif du sujet, il convient tout d'abord de réévaluer la chronologie dressée par Luc Martinez. Ce dernier affirme qu' « on ne peut diffuser du son de façon fiable et permanente que depuis l'avènement conjoint du support numérique et de l'informatique ", tout en concédant que "quelques réalisations pionnières aient précédé» (Martinez, p. 4). Un bref retour sur l'histoire du MEN amène à relativiser cet enchaînement: depuis le milieu des années 1950 jusqu'à celui des années 1960, le conservateur Jean Gabus a par exemple sonorisé tous ses projets d'expositions (plus de 7 au total ${ }^{1}$ ), notamment grâce à l'aide du fameux ingénieur Stefan Kudelski, ne craignant pas la bande magnétique pour diffuser commentaires, exemples musicaux ou ambiances sonores dans les murs de l'institution. Le système allait jusqu'à permettre de synchroniser l'éclairage des vitrines. Si l'ergonomie et la fiabilité étaient bien sûr précaires (les machines demeuraient capricieuses, les bandes fragiles; le déclenchement et le rembobinage des séquences nécessitaient un collaborateur dédié), l'effort témoigne d'une véritable politique d'intégration du matériel auditif à ce que notre illustre prédécesseur appelait le « musée dynamique » (Gabus 1956).

3 L'apparition des formats numériques n'a ainsi pas chamboulé d'un coup la situation: la voie était en partie balisée. En outre, malgré une souplesse d'usage bien supérieure, lecteurs $\mathrm{CD}$, ordinateurs, samplers et autres diffuseurs multipistes ont, jusqu'à une date récente, eu tendance à occuper beaucoup d'espace, à chauffer et à tomber régulièrement en panne, malgré la collaboration de plusieurs designers sonores. En termes de fiabilité, un palier notable n'a été franchi qu'avec l'apparition des modules PCB autonomes de type Movebox (premières utilisations entre 2008 et 2009 dans notre institution). Et encore... Si l'outil gère parfaitement la répétition et l'enchaînement de tâches complexes (son, vidéo, éclairage), c'est désormais la programmation et la compatibilité des fichiers qui peuvent entraîner des sueurs froides en amont.

Dans la pratique, nous avons donc assisté à une évolution, certes rapide mais graduelle, plutôt qu'à une révolution. Cette nuance peut sembler anecdotique à première vue mais invite à déconstruire le discours "technophile" qui accompagne systématiquement l'arrivée de nouveaux outils. Dans une perspective historique, sous l'effet conjugué du marketing et de l'enthousiasme, ceux-ci apparaissent toujours comme "révolutionnaires» et "impératifs», faute de quoi se profilerait le spectre d'un enlisement dont le musée incarne d'ailleurs souvent et ironiquement l'horizon. À titre d'exemple, pour justifier son projet de «Phonogramm Archiv », Carl Stumpf n'écrivait-il pas déjà en 1908: "Sans l'aide du phonographe, nous sommes plantés devant les vitrines du musée dans lesquelles sont conservés les instruments dans un bête silence d'outre-tombe, source d'émerveillement mais vides de sens. Ils demandent à être complétés d'urgence par une collection phonographique " (Simons, pp. 72-73) ? Par d'autres biais, la même injonction perdure à l'heure actuelle: saurait-il encore y avoir une ethnographie valable sans captation 
photographique, sonore ou filmique? Et à l'autre bout de la chaîne, sans installations idoines, les expositions peuvent-elles être autre chose qu'ennuyeuses et passéistes? Il s'agit là d'une spirale inflationniste dont les tenants et les aboutissants sont hélas peu débattus.

Sans nier l'intérêt que les technologies peuvent ajouter à une mise en scène, se pose néanmoins toujours la question du sens et de la fonction. Dans son article, Luc Martinez liste quatre domaines où le son peut se révéler particulièrement utile : relier ou dissocier certains éléments, signaler le parcours, étayer le propos ou s'afficher pour lui-même, en tant qu'expôt. Les deux premiers cas me semblent relever d'options scénographiques tandis que les deux suivants touchent plus directement au contenu de l'exposition. Dans tous les cas de figure, le sens demeure un enjeu central : en tant qu'éléments inhabituels et incontournables (sauf pour une diffusion au casque), les sons orientent fortement l'expérience d'un dispositif muséal. Leur choix relève ainsi du discours expographique, amenant à se poser la question de la marge de manœuvre qui peut être accordée à un designer sonore et du moment opportun pour intégrer un tel partenaire dans le processus créatif. Si Luc Martinez précise que «idéalement, un travail de conception sonore est à envisager dès la phase de définition d'un projet» (p. 5), ce beau principe élude nombre d'enjeux propres au champ muséal. Sans vouloir généraliser abusivement, l'expérience acquise au MEN m'amène à penser qu'une exposition s'accompagne toujours d'une longue phase de gestation où les contenus demeurent flous et se définissent par tâtonnements, dans un jeu de va et vient avec les intuitions de mise en scène. Si le regard d'un spécialiste peut s'avérer très profitable, il est toutefois orienté, poussant à favoriser son domaine de compétences plutôt qu'à évaluer sereinement l'opportunité d'y recourir (ou non) en fonction d'un propos basé sur une connaissance intime du sujet. Bref, l'inclusion précoce d'un designer sonore ne se résume jamais à optimiser la diffusion d'extraits, mais tend à plébisciter l'utilisation d'un média, le cas échéant avant même d'avoir élaboré un scénario valable. Un tel choix peut se justifier quand le thème entretient un rapport étroit aux archives ou à la musique, mais peut se révéler hasardeux, voire maladroit, en d'autres circonstances.

6 Indépendamment du concept s'esquissent également des questions plus générales et plus terre à terre : l'institution a-t-elle les moyens d'engager un ou plusieurs collaborateurs externes sur toute la durée d'un projet, de la conception à la réalisation physique ? Une telle requalification est-elle supportable à terme, sachant que d'autres corps de métiers la briguent également (scénographie, éclairage, graphisme, vidéo, mais aussi communication ou animation)? La multiplication des intervenants au stade de la conception n'amène-t-elle pas un risque d'enlisement au lieu de favoriser la prise de décisions qui seront de toute manière renégociées au stade de la mise en œuvre?

7 Il n'y a bien sûr pas de réponse générale et définitive à toutes ces questions mais elles méritent d'être posées face aux technologies et à leurs tenants qui - c'est un usage ancré de longue date comme l'a brillamment démontré Erika Brady (1999) - se vendent sous l'égide de la transparence, de la facilité et du progrès.

8 Dans sa deuxième partie, le texte de Luc Martinez dresse un panorama complet (et qui demeure très actuel) des outils à disposition pour éviter les effets de « bavures » sonores, comme si ce point constituait le principal obstacle empêchant l'intégration tous azimuts de pistes auditives au musée. À nouveau, penser par le biais de la technologie se révèle hasardeux. En effet, quelque 10 ans plus tard, la plupart des moyens évoqués ont fait la preuve de leurs avantages et de leur limites, sans radicalement changer la pratique de la 
muséographie : le haut-parleur directionnel n'est pas aussi précis qu'escompté (du moins celui que l'équipe du MEN a pu tester dans le cadre de l'exposition La marque jeune $e^{2}$ ) et ne se prête guère à diffuser autre chose que des voix (Luc Martinez est déjà clair sur ce point) ; les transducteurs de surface peinent à convaincre malgré leur concept séduisant (les vitrines sonores du dépôt d'instruments de musique au musée du Quai Branly en sont un fameux exemple); la diffusion multipiste peut avoir des applications de nature théâtrale mais n'a guère de sens pour présenter des sources archivistiques; les moyens d'isolation et de traitement acoustique supposent des contraintes et des moyens qui les rendent surtout envisageables à l'occasion de rénovations ou d'aménagements à long terme (un deuxième cas à rebours de la souplesse que recherchent de plus en plus les institutions culturelles). Rétrospectivement, il faut noter qu'au cours des dernières années, de nombreux musées ont tourné le dos à l'usage de sons ouverts, préférant miser sur une réinvention de l'audioguide via les supports mobiles qu'offrent tablettes et smartphones. Nouveaux outils, nouvelles utopies d'interaction, de synthèse multimédia, de personnalisation et de délocalisation de l'expérience muséale (le fameux poncif d'une visite à la carte, dont certains aspects pourraient être gérés ou vécus à domicile) et souvent, à la clef, nouvelle répétition des travers que Luc Martinez brocarde au sujet des anciennes générations, soit une tendance à réunir trop d'informations, ce qui a pour effet de lasser le visiteur et/ou de le détourner de la mise en scène physique ${ }^{3}$.

9 À ce stade, le lecteur en viendra sans doute à se demander ce qu'il faut retenir de ma démonstration: exposer le son est-il une cause perdue et les progrès techniques un miroir aux alouettes? Je serais navré de donner cette impression car, au fond, je partage l'analyse de Luc Martinez, à savoir que le son offre un riche éventail de possibilités en termes de dramaturgie et de transmission; un éventail qui est encore largement sousexploité au sein des musées. Mon point de vue diverge concernant les manières de changer cette donne, estimant que les techniques ne constituent pas des solutions en elles-mêmes. Vues sous cet angle, elles se révèlent coûteuses et contraignantes, soumettant les contenus à des logiques d'usages rapportées. Si, comme nous le défendons au MEN, l'exposition est un dispositif qui mobilise divers médias au service d'un propos intégré, à chaque fois unique, il convient alors de réfléchir non plus en seuls termes d'efficacité mais aussi de cohérence, d'originalité, souvent à rebours des usages clef en main et de la perfection vantée dans les modes d'emploi.

10 C'est précisément ce que nous avons fait dans le cadre des expositions Bruits et HorsChamps, qui exploraient respectivement la manière dont le son et l'image ont été mobilisés par les chercheurs en sciences sociales depuis l'apparition du phonographe et de l'appareil photo. Dans les deux cas, la technologie n'était pas seulement un prétexte à soumettre des exemples mais une piste narrative centrale, interrogeant les rapports entre médias, production scientifique, vie sociale, construction des rapports à l'Autre et au passé. L'habituel clinquant des discours technophiles était ainsi passé à la moulinette de juxtapositions inhabituelles (par exemple tous les principaux modes de diffusion sonores, des plus ouverts au plus fermés), de télescopages historiques (séquences rotoscopiques et jeu vidéo pour interroger la captation du geste en anthropotechnologie), d'hérésies conceptuelles (assumer l'empilement cacophonique d'extraits musicaux dans certaines zones pour dire la relativité des principes esthétiques) ou de délires poétiques (machines à congeler le patrimoine ou à distiller l'immatériel pour brocarder les travers du geste archivistique). L'avantage de ces solutions était triple : transcrire le propos dans 
l'espace, illustrer ses enjeux bien mieux qu'à travers un appareillage high-tech et rester économiquement accessibles.

11 Pour développer ce genre de projet, nous avons la chance de pouvoir compter sur une équipe de réalisation externe dont les membres et les compétences variées (elle comprend bien sûr un designer sonore) peuvent être mobilisés en fonction des besoins, sans obligations ni besoin de prouver quoi que ce soit. La situation est très agréable en ce sens qu'elle permet à chacun d'intervenir plus ou moins tôt, en fonction de l'évolution du scénario, selon une logique qui est à la fois participative et centralisée. Elle permet de surcroît une étroite collaboration entre les conservateurs et les techniciens/designers, poussant chacun à réfléchir hors de ses habitudes.

En guise de conclusion, plutôt que de souhaiter l'avènement de nouveaux outils « spécifiquement élaborés pour assister la gestion et la diffusion du son au sein d'une mosaïque multimédia de plus en plus complexe et connectée » (Martinez, p. 12), je me permettrais donc plutôt d'espérer la généralisation d'échanges aussi francs et décomplexés que possible entre muséologues et designers sonores. C'est de leur entente et non de leurs outils que peuvent naître des perspectives d'exposition intéressantes.

\section{BIBLIOGRAPHIE}

Brady, E. A Spiral Way: How the Phonograph Changed Ethnography. Univ. Press of Mississippi, 1999, $156 \mathrm{p}$.

Gabus, J. Musée statique et musée dynamique, Revue Romande des Arts, n 30-31, 1956, pp. 5-12.

Sierro, V. Le musée dynamique de Jean Gabus (1955-1978), in Gonseth, O., Hainard, J. et Kaehr, R/ (dir.) Cent ans d'ethnographie sur la colline de Saint-Nicolas 1904-2004. Neuchâtel : MEN, 2005, pp. 343-366.

Simons, A. (éd.) Das Berliner Phonogramm-Archiv 1900-2000. Sammlungen der traditionellen Musik der Welt. Berlin, 2000.

\section{NOTES}

1. Brésil : de la plume au gratte-ciel (1955) L'art artisanal de la Chine (1956), Sahara ‘57 (1957), Bulgarie : 2500 abs d'art (1958), À quoi jouent les enfants du monde ? (1959), Parures et Bijoux dans le Monde (1961), La Main de l'Homme (1963) ainsi que la salle d'exposition permanente consacrée à la Mauritanie.

2. La marque jeune, au MEN, du 28 juin 2008 au 15 mai 2009

3. Ma première visite avec tablette a eu lieu au musée national suisse de Zurich, dans le cadre d'une exposition consacrée au chanteur Mani Matter en 2011. L'expérience s'est vite révélée frustrante : longue file d'attente pour obtenir un Ipad configuré (pour des questions de droit d'auteur, l'application ne pouvait être chargée à domicile) ; impression générale que la mise en scène physique avait été négligée au profit d'une balade virtuelle fondée sur la répétition des 
mêmes contenus, dans une version augmentée (plus d'images, de films, d'extraits sonores) mais sans offrir de nouvelles grilles de lecture.

\section{RÉSUMÉS}

À l'occasion des 30 ans de l'OCIM, La Lettre de l'OCIM propose à ses lecteurs, dans chaque numéro de l'année 2015, un retour sur un article marquant de l'histoire de la revue. À partir d'un corpus d'articles choisis par la rédaction de La Lettre de l'OCIM, les membres du comité des Publications de l'OCIM ont sélectionné plusieurs contributions.

Dans cette perspective, il a été demandé à l'auteur ou à un expert du domaine de revisiter la problématique exposée dans l'article à la lueur des changements intervenus - notamment dans les pratiques professionnelles - depuis son écriture et de proposer des éléments prospectifs sur la question.

À la lueur de son expérience au musée d'Ethnographie de Neuchâtel, l'auteur réexamine la problématique de la mise en son des espaces muséaux abordée par Luc Martinez dans l'article «Oyez! Le son s'expose » publié dans le nº 107 de La Lettre de l'OCIM de septembre-octobre 2006, en posant la question du sens et de la fonction et de l'échange entre le muséologue et le designer sonore.

INDEX

Mots-clés : musée, son, musique

\section{AUTEUR}

\section{YANN LAVILLE}

conservateur adjoint au musée d'Ethnographie de Neuchâtel

Yann.Laville@ne.ch 